\title{
Nivel medio: encuentros y desencuentros entre el enseñar y aprender
}

\author{
Marta Florencia Herrera \\ María Elena Ponzoni \\ Patricia Inés Barraza \\ (Universidad Nacional del Comahue. Argentina)
}

\section{Resumen}

El presente artículo se construye a partir de los avances que realizamos en el proyecto de investigación: "¿Qué enseña la escuela media? Un estudio de sus huellas en la voz de sus egresados". Como tal, el mismo no plantea conclusiones finales, sólo algunos interrogantes que han dado origen a nuestra investigación, como así también, aquellos indicios en torno a un tema tan debatido como los son las huellas o surcos que la escuela media deja en los egresados.

Reflexionar sobre las relaciones del docente con el conocimiento y los alumnos, en términos de huellas, espejos o señales es una problemática que nos permite analizar diversos aspectos de nuestras prácticas educativas, en especial, la posibilidad de cuestionar aquellas supuestas verdades esenciales en torno a la enseñanza y el aprendizaje.

Las interpretaciones acerca de las expresiones valorativas de los egresados nos posibilitó reconocer algunas características de la impronta de la enseñanza en el nivel medio, entre ellas, la distancia entre los tiempos del enseñar y el aprender, la forma del trabajo con el contenido, la capacitación docente, el lugar de integración de nuevas tecnologías, las modalidades de enseñanza, los aspectos comunicacionales y el sentido de pertenencia.

\section{Palabras clave}

Enseñanza - Aprendizaje - Valoraciones - Escuela Media - Huellas.

\section{Summary}

This article is based on the progress we made in the research project: What teaches middle school? A study of the footprints in the voice of their graduates. As such, it poses no conclusions, just some questions that have led our investigation, as well as those topics that have been debated about graduates of middle school. 
Think about the relationships of teachers with the knowledge and the students in terms of tracks, signals or mirrors is a problem that allows us to analyze various aspects of our educational practices, in particular, to question alleged essential truths relating to teaching and learning.

The interpretations about the value of graduates allow us to recognize some characteristics in the imprint of teaching at the secondary level, including the distance between the times of teaching and learning, the way of working with the content, training teacher, integrating new technologies, modes of teaching, communication aspects and the sense of belonging.

\section{Key words}

Teaching - Learning - Valuations - Middle School - Footprints.

\section{Nivel Medio: encuentros y desencuentros entre el enseñar y aprender}

El presente trabajo se desprende de los avances realizados en el proyecto de investigación: “¿Qué enseña la escuela media? Un estudio de sus huellas en la voz de sus egresados", que tiene como objetivo general interpretar las huellas de la enseñanza que deja o propone la escuela media en el Alto Valle de Río Negro. En los objetivos específicos, trataremos de reconocer situaciones, experiencias y acontecimientos de la escuela media para interpretar los sentidos y significados otorgados a ese nivel por los sujetos que lo han transitado. El universo de análisis está constituido por egresados que tengan entre cinco y diez años de finalización de la escuela media, quienes se encuentran cursando estudios superiores, universitarios y no universitarios. El instrumento elegido para la recolección de los datos fue la entrevista semiestructurada, porque permite, por un lado, indagar sobre aquellas cuestiones que le interesan al investigador en los distintos modelos de recuerdo y por el otro, permite que salgan a la luz aspectos interesantes no previstos inicialmente.

Los datos obtenidos posibilitaron problematizar algunas cuestiones con relación a la enseñanza y el aprendizaje, a partir de las expresiones vertidas por los entrevistados acerca de la huella o impronta que les produjo su paso por la escuela media.

Entendemos que la huella cognitiva no es pasado sino presente y a través de las narrativas es posible identificar encuentros y desencuentros entre ambos procesos.

Dar la voz a los egresados e interpretar sus aseveraciones nos permitió analizar las discontinuidades que ellos retoman como importante o 
desafiante en sus aprendizajes al encontrarse transitando el sistema universitario. Esta pausa (período de tiempo que creemos lleva consigo una reflexión imperante) le permite, a los estudiantes, descentralizar, expresar y recrear aquellas experiencias que consideran destacables -o no- de la escuela media.

Para Winicott (2004) el jugar tiene un tiempo y un lugar que no pertenece a la realidad psíquica ni a la realidad exterior, sino a una "zona transicional". Jugar es hacer y el juego es en sí mismo terapéutico. En él, niños y adultos están en condiciones de ser creadores.

Creemos que el poder "volver" sobre las vivencias pasadas en sus años escolares les permite a los egresados retomar este "juego" que, como lo expresa Winicott, es terapéutico en la medida que el adolescente reconstruye sus propios pasos y lugares construidos.

Es decir, este re-volver a aquello que se reconoce como un jugar, viabiliza repetir, reaccionar y responder a lo nuevo como un itinerario dialógico. Esto le concede reconstruir su historia escolar y narrar qué valora -o no- en el ahora. Al retomar su voz como ex alumno de la institución comprende su recorrido escolar, su historia y la influencia de aquellos que compartieron esa experiencia junto a él (profesores, compañeros, directivos...). Esta posibilidad de agencia y autovaloración de los sujetos escolarizados, ya señalada por Bruner y analizada en otros escritos (Herrera y Barraza, 2008) acentúa los rasgos señalados.

Los egresados rescatan a los docentes como personajes centrales, no sólo en relación con sus aprendizajes académicos, sino como personajes que conformaron gran parte de su identidad social e ideológica.

Esto nos lleva a pensar cómo la idea de construcción del conocimiento en el diseño de las clases implica considerar las teorías implícitas que los docentes tienen acerca de cómo enseñar, acerca de cómo aprenden los alumnos, y acerca del conocimiento. En este marco, diversas investigaciones proponen la práctica reflexiva y la narración de las interpretaciones de aquellas pautas escolares que son consideradas "buenas" razones en las lógicas de los aprendizajes. Estas pautas, señalizadas en las voces de nuestros egresados conforman surcos interesantes de analizar.

En esta interpretación, las narrativas de los procesos de enseñanza y aprendizaje no han de entenderse como algo definitivamente dado, sino que se constituyen dinámicamente, mutuamente, con la actividad de los participantes.

Bárbara Rogoff ha aportado el concepto de participación guiada para entender cómo se produce el aprendizaje y el desarrollo cognitivo en las diferentes edades escolares; remarcando que la misma no posee nunca un 
sentido individual, aún cuando a veces las relaciones adulto-adolescente se produzcan de tú a tú:

"El concepto de participación guiada incluye tanto el papel que desempeña el individuo como el contexto sociocultural. En lugar de intervenir como fuerzas separadas o que interactúan, los esfuerzos individuales, la interacción social y el contexto cultural están intrínsecamente enlazados a través de todo el desarrollo infantil, hasta que los niños llegan a participar plenamente en la actividad social" (Rogoff, 1993, p. 43).

De la misma forma, podemos hacer referencia al carácter dialéctico de la apropiación de las herramientas culturales por parte de los aprendices, pues éstos, a la vez que aprenden a utilizarlas (se las apropian), pueden modificarlas o usarlas de nuevas maneras. Bruner afirma el concepto de "aprendizaje por descubrimiento", que se comprende dentro de un enfoque negociador de la educación:

"No se trata sólo de que el niño deba apropiarse del conocimiento, sino que debe apropiarse de él en una comunidad formada por aquellos que comparten su sentido de pertenencia a una cultura. Es esto lo que me hace subrayar no sólo el descubrimiento y la invención sino la importancia del negociar y el compartir, en síntesis, de la creación conjunta de la cultura como objeto de la enseñanza y como paso adecuado para llegar a ser un miembro de la sociedad adulta en la cual pasamos nuestra vida" (Bruner, 1988, p. 132).

Por su parte, Lacasa, Cosano y Reina se basan en Cole (quien afirma que la cultura y el conocimiento se generan mutuamente en la Zona de Desarrollo Próximo), y, será a partir de estas relaciones socio-cognitivas, donde los estudiantes pueden desarrollar aspectos que superen la mera práctica de aprendizaje académico, retomando aspectos que conllevan la cultura misma de la escuela., como son la idea de grupalidad y de cooperación. Todo ello favorecido por prácticas de enseñanza que se basan en tareas guiadas y cooperativas entre el estudiante, el docente y el medio cultural. Estos aspectos, quizás ya reconocidos por todos nosotros, conforman las prácticas que años más tarde son destacadas por los egresados.

"La cultura crea el conocimiento en la zona del desarrollo próximo estructurando las prácticas en las que los adultos comprometen a los niños para favorecer su desarrollo. El conocimiento crea cultura organizando la interacción social entre el niño y los mayores, ya que juntos generan nuevas 
prácticas o ideas que serán aceptadas en el grupo social" (Lacasa, Cosano y Reina, 1997, p. 10).

Por ello, las actividades y los modelos didácticos son constructos desde los cuales se crea la posibilidad de innovación en las teorías de las cogniciones y la enseñanza guiada. Las contribuciones activas de los individuos, los compañeros, las tradiciones, las demandas cognitivas y los materiales escolares son inseparables de las condiciones que se dan dentro del proceso de enseñanza y de aprendizaje.

Fenstermacher (1989) (1) señala que la enseñanza crea unas condiciones sociales en las que el alumnado debe asumir su rol y realizar las tareas que éste requiere, tareas que son objeto de un aprendizaje constante y dinámico.

\section{La escuela media: aspectos medulares de las prácticas}

La escuela media conforma una estructura determinada del sistema educativo argentino para adolescentes y jóvenes. Su régimen de trabajo genera demandas cognitivas específicas, tales como el pensamiento científico, la construcción de la subjetividad y la autonomía, la apropiación del conocimiento disciplinar, entre otras.

En las entrevistas realizadas, los egresados efectúan reconocimientos y críticas al rememorar su paso por la escuela media y estas valoraciones muestran aspectos medulares de las prácticas, que refieren tanto a sus propias prácticas de aprendizaje, como en relación al resto de los estudiantes y de los docentes. No resulta novedoso afirmar que la cotidianeidad de las prácticas en las aulas está teñida de conflictos y desencuentros entre la enseñanza de los docentes y el aprendizaje de los alumnos, tal como hacíamos mención en el escenario de la rutina de nuestro anterior proyecto de investigación.

Conflictos y desencuentros que, en la voz de los entrevistados aparecen como cuestionamientos hacia las propuestas de enseñanza, en el sentido de la cantidad de temas que se ven, el poco tiempo que se le dedica a cada uno y la necesidad de hacerlos o presentarlos de manera interesante:

“... eran muchos temas... me parece que es preferible dedicarle más clases a un tema y bien que muchos temas..." (E. 10).

“... relacionar, ver películas, estimular, usar el arte...” (E. 13).

“Daría más práctica. Los contenidos relacionados con la vida real..." (E.15). 
"Lo podrían cambiar o podrían poner algo más interesante, más actual o encararlo de otra forma" (haciendo referencia a los contenidos de: matemáticas, física y química) (E. 9).

Estos cuestionamientos tienen que ver, por un lado, con la distancia existente entre los tiempos de la enseñanza y los procesos de aprendizaje $y$, por otro lado, con las diferentes formas de trabajo con el contenido. Con respecto al primero, dichos cuestionamientos constituyen una fuente de problematización de la práctica de los docentes que se convierten en incidentes críticos (2) que demandan ser significados.

En los ejemplos citados, los temas enseñados, recordados a la distancia, aparecen como excesivos, rutinarios y con escaso margen de tratamiento. Como contrapartida, en nuestra anterior investigación, ante las dificultades de aprendizaje, las intervenciones docentes están dirigidas -casi exclusivamente- a las modificaciones en las estrategias y/o recursos de orden metodológico. En esos relatos, los docentes sostenían que las insuficiencias en el aprendizaje estudiantil emergen acompañadas de diversas hipótesis acerca de su origen y es caracterizado con particularidades específicas. El origen de las dificultades de aprendizaje queda generalmente vinculado a condicionantes de tipos socio-culturales propios de esta época, tales como el descrédito del conocimiento como determinante de ascenso social, de las instituciones escolares como referentes claves de la cultura y de los profesores como autoridades epistémicas y morales. Las dificultades para aprender se describen como ausencias o insuficiencias de procesos cognitivos básicos, de carácter subjetivo e individual, que es necesario activar o corregir para que la enseñanza de contenidos disciplinares pueda encontrar un legítimo lugar de participación.

Así, los docentes entienden que sus intervenciones deben convertirse en prácticas de reparación o compensación de desajustes en procesos tales como la atención, la motivación y/o el razonamiento de los alumnos. Estas prácticas son vivenciadas como imprescindibles, pero, al mismo tiempo se señalan como suplementarias, adicionales o yuxtapuestas en relación a su tarea específica de enseñar contenidos disciplinares. Puede interpretarse que los docentes entienden que tales procesos son una condición que los alumnos deberían poseer como capital personal adquirido en el nivel primario, como prerrequisito del aprendizaje y no como procesos que se aprenden en comunidades de prácticas especificas, como es el caso de las aulas del nivel medio.

Resulta evidente que existe un esfuerzo por superar las propuestas por parte de los docentes pero que en el recuerdo de los egresados esos intentos no han quedado registrados. Por el contrario, reclaman, de algún 
modo, una reparación en los aprendizajes de los actuales alumnos. Podríamos pensar que esta fuerte valoración tiende a propiciar otros aprendizajes genuinamente transformadores (3), en la búsqueda de alternativas para resolver las dificultades que se presentan. El camino es el del conocimiento y en él se instalan las tecnologías: sean tradicionales o austeras como las del pizarrón, atractivas y producidas para otros fines como los films; sofisticadas como las que nos puede proveer un programa de actividades para la enseñanza de un tema específico del currículo y poner de manifiesto la enseñanza en su dimensión moral que llamaremos conciencia expresiva (Litwin, 2008).

Como habíamos planteado anteriormente, los egresados cuestionan no sólo los tiempos de enseñanza sino también el trabajo con el contenido. Al respecto, entendemos por trabajo con el contenido como la intencionalidad del docente en la selección y jerarquización del conocimiento a ser enseñado, como así también, las actividades propuestas para ello. Desde esta perspectiva proponen formas más innovadoras en las disciplinas de matemática, física y química o la incorporación de asignaturas más humanísticas en la formación técnica.

Estas expresiones dan cuenta de la necesidad de un cambio o reconstrucción de los marcos de referencia que poseen los docentes acerca de las diversas formas y tiempos que adoptan las propuestas de enseñanza. El cuestionar dichas propuestas constituiría, sin lugar a dudas, una demanda potente y relevante hacia el nivel medio educativo, pero al ser expresiones de un pasado escolar no pueden ser satisfechas en la actualidad. Sin embargo, resultaría de interés contar con este tipo de demandas a la hora de plantear las propuestas de enseñanza por parte de los docentes en ejercicio. En palabras de Maxine Greene (2005, p. 322): "Abordar la enseñanza y el aprendizaje de ese modo es interesarse por la acción, no por la conducta. La acción implica emprender iniciativas; significa moverse hacia un futuro contemplado desde el punto de vista del actor o el agente".

"Me gustaría estudiar y aprender las cosas con más tranquilidad porque es como que el tiempo me apura..." (E: 24).

"Una de las cosas que más me costó en la universidad era el tema que es otro ritmo..." (E: 25).

Los egresados demandan o requieren diferentes ritmos de estudio, dado que al rememorar observan que los tiempos debieron ser diferentes para favorecer sus aprendizajes, tanto en el nivel medio como en el superior. 
Por otra parte, hacen referencia a otros aspectos relacionados con la actividad docente, tales como la capacitación y la integración de nuevas tecnologías, ya mencionadas en ejemplos anteriores:

“... yo creo que la capacitación con las cosas que hay hoy en día, con todos los avances de la tecnología... creo que sería una muy buena forma de enseñar lo mismo, pero de otra manera..." (E.9).

“... se tendrían que especializar año tras año...” (E. 21).

Resulta claro que algunas de las propuestas de enseñanza vividas por los entrevistados, ofician de verdaderos obstáculos para sus aprendizajes. Es decir, pareciera que cualquier cambio significativo por parte de los docentes implicaría la revisión y transformación de las prácticas, pero tamaño emprendimiento requiere que el docente se encuentre con sus propios límites dado que es, generalmente, una tarea solitaria producto de la fragmentación y la balcanización propia de la escuela media (Hargreaves, A., 1996; Pérez Gómez, A., 1998).

Sabemos que la falta de osadía docente o la imposibilidad real de actuar son dos procesos diferentes y es necesario distinguir las condiciones en las que se producen uno u otro. Así, la reflexión y las alternativas en torno a ciertas problemáticas sociales e institucionales son comprometidas y críticas, pero, la fuerza de lo instituido, la desconfianza a lo novedoso, y la exigencia de un trabajo en soledad constituyen verdaderos impedimentos para la actuación. Estos obstáculos no son productos artesanales propios de los docentes, sino que su construcción se elabora, en gran medida, en la lógica institucional y las demandas sociales. Ahora bien, a pesar de esta fragmentación, reflejo de las condiciones sociales actuales, los docentes asumen grandes desafíos y compromisos reveladores de buenas prácticas. En nuestra anterior investigación, publicada bajo el título "Evaluar la propia enseñanza. Los escenarios de la escuela media” (2007), sosteníamos que esos esfuerzos daban cuenta de transformaciones fructíferas para la enseñanza y mostrarían un guión para la construcción del oficio que se desplazaría entre el ensamble y la yuxtaposición de propuestas didácticas (4). Los egresados rescatan estas prácticas innovadoras:

"En Plástica veíamos los diferentes movimientos artísticos y luego hacíamos trabajos prácticos, con imágenes, con recortes y nos enseñaron a dibujar, los colores, la paleta de colores, la perspectiva." (E: 15). 
"Con la de Lengua pasaba lo mismo. Yo la escuchaba atentamente cuando hablaba y pensaba: ¡cómo sabe esta mina! Nos presentaba la novela y nos hacía ver la película para que pudiéramos diferenciar el texto escrito del guión. Vimos Plata quemada y leímos el libro. Luego nos hacía debatir sobre eso y teníamos nuestras posturas. Eran muy interesantes esos debates. $Y$ nos obligaba a leer..." (E: 25).

Estas experiencias en los recuerdos aluden a la transferencia de habilidades generales, habilidades específicas y contenidos disciplinares. Dichas alusiones iluminan el concepto de andamiaje en la participación guiada de los docentes. Bruner (1997) define dicho proceso como “... las formas en que un adulto puede colaborar con el aprendizaje de un niño, especialmente en relación a su capacidad para adaptarse a los cambios y generar acciones capaces de resolver las problemáticas o situaciones que le corresponde vivir...". En este mismo sentido, los egresados realizan señalamientos que se relacionan con los aspectos comunicacionales dentro y fuera de la institución.

"integrarme más... integrarme y también mejoraría la charla con los profesores, tener más relación...” (E. 12).

"Disfrutar más con mis compañeros o trabajar más en grupo..." (E.13).

Estos enunciados estarían ligados a la construcción de lazos comunicacionales más firmes, ya que el lenguaje es una "herramienta cultural esencial", porque la utilizamos para compartir la experiencia y para darle sentido colectiva y conjuntamente. Al decir de Mercer (1995, p.15) "el lenguaje es, por lo tanto, no sólo un medio por el cual los individuos pueden formular las ideas y comunicarlas, sino que también es un medio para que la gente piense y aprenda conjuntamente". Así, el lenguaje es un medio para transformar la experiencia en conocimiento y comprensión culturales.

La integración grupal se vuelve un factor fundamental durante la adolescencia y obliga a gestionar pedagógicamente un proceso de cambio en la composición social de las ideas del profesorado que considere y que aproveche la subjetividad juvenil del Nivel Medio. Esto constituiría, evidentemente, uno de los máximos desafíos de las expectativas en la planificación social del conocimiento. Es decir, pensar propuestas de enseñanza que contemplen los agrupamientos, el apoyo y la integración, como cruce científico curricular en las construcciones de la cultura y de las identidades. Construcciones en las que el tiempo de la enseñanza y el 
tiempo del aprendizaje son ideas intersubjetivas, pero podrían considerarse como experiencias inéditas de posibilidad didáctica.

Otras expresiones aluden a mirar las cosas como si pudieran ser de otro modo, lo que implicaría la "búsqueda de certezas" ante la posibilidad de reestructurar o reformar los viejos modelos escolares. Maxine Greene sostiene que, tanto para aprender como para enseñar, se ha de tener conciencia de dejar algo atrás y de perseguir algo nuevo, y esa clase de conciencia deber ir ligada a la imaginación. La imaginación, según Dewey (1934, p. 272) constituye la puerta de entrada por la que los significados de experiencias pasadas llegan hasta el presente y resultan "el ajuste consciente entre lo nuevo y lo viejo".

"... hay que buscar entendimiento entre profesores y alumnos... hacer talleres, algo, donde se puedan poner cosas en común" (E. 22).

“... para que el día de mañana, los chicos que salen de 5to. año no salgan con que la vida es color de rosas... para abrir la cabeza a los chicos..." (E. 9).

Además, en estos ejemplos, los egresados marcan la necesidad de establecer redes de entendimiento entre docentes y alumnos. Unas tienen que ver con la búsqueda de objetivos comunes que optimicen los aprendizajes. Otras, con la búsqueda de discursos que permitan mayor reflexión de la realidad social que les toca vivir.

Otro aspecto a considerar es la importancia que le otorgan los egresados a algunas interacciones en la dimensión colectiva del dispositivo escolar que les demandaba la escuela media. Por ejemplo, el rol de los centros de estudiantes, que se originan como agrupamientos entre pares en respuesta al interés de los alumnos, posibilitando modos de participación, aportes e integración de actividades programáticas o extraprogramáticas en la unidad escolar. Este tipo de agrupamiento de los estudiantes tiene su origen en la escuela media y se continúa en el nivel superior, pero que muchas veces su accionar excede los ámbitos institucionales y resulta cuestionado y resistido:

"La escuela debería tener más autoridad ante los alumnos, no permitir que los alumnos tengan tanta autoridad (Centro de Estudiantes)" (E. 19).

Estos espacios de agrupamiento podrían favorecer u obstaculizar los procesos cognitivos pero, a. su vez, generan huella en la toma de perspectiva y el descentramiento de procesos individuales. 
"Disfrutar más con mis compañeros o trabajar más en grupo..." (E.13).

La idea de grupalidad como un modo de garantizar el desarrollo del pensamiento formal de la cognición debería tener su correlato con el espacio físico de las instituciones escolares y que, la mayoría de las veces no resulta el más apropiado o requerido:

"cambiaría el sistema de las aulas.... el patio, porque la verdad que no se aprovechó mucho, habiendo tanto lugar afuera para hacernos un espacio a nosotros para estar y nunca se aprovechó ese espacio y quizás si hubiesen hecho algo como para entretenernos por lo menos en los recreos, hubiese sido más... más bueno, poner música, qué sé yo, en los recreos, porque hay varias escuelas que tienen ese sistema y nosotros nunca tuvimos esa oportunidad" (E. 23).

Cabe señalar que el egresado de la escuela industrial manifiesta fuertemente la necesidad de fomentar o promover el sentido de pertenencia a la institución, reconocimiento que no aparece en las expresiones de los otros egresados. Esto daría cuenta de una modalidad institucional que favorece actitudes subjetivas en los egresados que, a la distancia, son vistas como necesarias e importantes.

En diversas investigaciones se plantea "el sentimiento" de pertenencia de las escuelas técnicas como un aspecto notable al momento de pensar en la formación específica de dichas instituciones. Esto se lo puede alegar a diversos factores, desde la mayor carga horaria que poseen, como así también a los vínculos personales que se establecen entre los docentes y alumnos al participar en mayor número de actividades debido a los talleres y horas extraescolares. Sin embargo, la distinción presente en el desarrollo de este sentimiento de pertenencia o compromiso entre alumnos egresados de las escuelas medias comunes y los alumnos de las escuelas técnicas nos obliga a formular algunos interrogantes: ¿Es posible que en estas escuelas los estudiantes desarrollen formas de trabajo cooperativo más personales?

Las diferencias notables entre las escuelas, ya sea por la carga horaria o la "distinción" histórica de la escuela técnica, ¿puede provocar que los estudiantes desarrollen con mayor fuerza este sentido de pertenencia y compromiso hacia ese tipo de escuela?

“... los estudiantes se sienten comprometidos con la institución, son parte de y para ella. Esta cualidad de la pertenencia, traspasa 
los límites de las escuelas regionales y se extiende, según testimonios de los propios profesores y alumnos a todas las técnicas del país..." (Martín, 2001, p. 117).

De ser así, deberíamos repensar en los análisis realizados con relación a los procesos de andamiaje que se establecen dentro de la institución, los cuales, indefectiblemente, conmueven y "marcan" las huellas de los egresados.

\section{A modo de reflexión}

Analizar las huellas de la enseñanza a través de las propias voces de los egresados nos posibilita abrir un nuevo debate en torno a las tramas didácticas en términos de buenas o malas razones, de encuentros y desencuentros, de reclamos y demandas, en torno a temáticas específicas de enseñanza, que muchas veces como docentes no destacamos en nuestras prácticas formales.

“... las experiencias pasadas, su análisis y sus significados [...] liberan a la imaginación y permiten una visión enriquecida del pasado. El esfuerzo de dar forma de narración a las experiencias vividas también crea sentidos y nos brinda la posibilidad de recuperar historias, tradiciones, maneras de pensar y obrar..." (Litwin, 2008, p. 19).

La trama didáctica implicada en los relatos nos pone en dialogicidad con los conceptos de andamiaje y la distribución social del conocimiento a través de los contenidos como hechos, conceptos y valores distribuidos.

La construcción del conocimiento en el diseño de la enseñanza implica considerar las teorías implícitas que los docentes tienen acerca de cómo enseñar, acerca de cómo aprenden los alumnos, y acerca del conocimiento. En este marco, distintas investigaciones proponen la práctica reflexiva con escenarios donde la motivación y las estrategias de enseñanza en los nuevos protagonistas se re-visitan en la pausa de los egresados con el contenido y desde la validación de las metodologías. El tiempo curricular de la enseñanza y la subjetividad pedagógica del aprendizaje se conjugan de modo tal que el pasado constituye el sentido del presente en una relación del egresado con el contenido y la propuesta de enseñanza. Esta relación nos posibilita valorar prácticas reflexivas donde la clase y los de la clase planteen procesos de convergencia en el andamiaje social, a pesar de los encuentros y desencuentros producidos.

La participación guiada y la dimensión comunicativa de la enseñanza como innovaciones en las tramas didácticas de la escuela media, como lo 
son los agrupamientos o las comprensiones implicadas en la clase, nos permiten visualizar los modelos de actividad construidos desde el aprendizaje. Por supuesto, deberíamos referirnos a distintos modelos y sus derivaciones conceptuales, entendiendo que cada modelo jugó y aún lo hace, un rol bastante definido en distintos momentos de la historia, adaptándose o no, según los casos, a las situaciones particulares de cada región. Lo que siempre se percibe en ellos, y más aún en el encanto de la enseñanza, son las ilusiones y las perspectivas de desarrollo para el cambio, por qué, sino y desde dónde. Éste será nuestro desafío.

Por último, citamos a Elliot W. Eisner (1982) "¿Qué ofrecemos a los estudiantes y cuál será la base de nuestra selección? Es preciso atender a la enseñanza. Ningún currículum puede sobrevivir a la incompetencia docente; pero tampoco vale la pena enseñar bien un currículum malo".

\section{Notas Bibliográficas}

(1) Fenstermacher, G. (1989). "La integración requerirá una gran sensibilidad para comprender los factores que, en situaciones concretas, exigen un delicado equilibrio entre sus valores, sus propósitos y sus acciones. No hay recetas infalibles y puede ocurrir que no siempre las cosas salgan como uno desea. Por supuesto, usted puede decidir, con buenas razones, que las ventajas de adoptar una de las perspectivas exceden con mucho las desventajas de intentar la integración de las tres. Su elección de una visión normativa de la enseñanza debería basarse en su mejor juicio personal".

(2) "Un incidente crítico es un evento que puede ser llamado a conciencia fácil y rápidamente porque es recordado vívidamente. Consideramos a los eventos críticos significativos porque son inesperados -nos toman por sorpresa-" (Brookfield, 1997, p. 23). El autor aclara que estas experiencias a veces son maravillosas, a veces desmoralizantes, y frecuentemente implican una mezcla de ambos sentimientos.

(3) Es necesario, en principio, diferenciar el aprendizaje transformador del aprendizaje informativo. Siguiendo a Kegan (2000), acentuamos que el aprendizaje 'informativo' se refiere a la acción de rellenar una forma ya existente. Mientras que el aprendizaje 'transformador' pone en riesgo de cambio la forma misma, aunque esos cambios no necesariamente impliquen incremento de la capacidad. El corazón de la forma a la que se refiere Kegan es un camino de conocimiento, lo que Mezirow denomina 'marco de referencia'. "Un marco de referencia es una "perspectiva de significado", la estructura de los supuestos y expectativas a través de los cuales se filtra la experiencia" (2000, p. 16) pero a su vez se forman como resultado de vías de interpretación de la experiencia socialmente construidas. Si bien pueden ser implícitos o explícitos, las más arraigadas creencias sobre la propia subjetividad o sobre el mundo son inferidas a partir de lo empírico y permanecen fuera de la conciencia. Esos 
marcos pueden generar creencias y opiniones que provean justificaciones para guiar la acción. Es así, que un genuino aprendizaje transformador implica, para el docente, la reconstrucción de sus marcos de referencia para hacerlos más abarcativos, integrados y capaces de cambios que involucren la subjetividad.

(4) Cabe aclarar que no sólo las prácticas autoevaluativas son inherentes al aprendizaje del oficio docente, sino que se despliegan en diferentes escenarios cuyas tramas dan cuenta de proyecciones ideológico-epistémicas, políticas educativas y representaciones sociales, como así también, procesos comunicacionales e intersubjetivos que orientan epistemológica y moralmente a sujetos concretos. En tal sentido, hemos reconocido tres escenarios que muestran aspectos centrales de la dinámica en la que se aprende el oficio de enseñar. Estos escenarios son: el de la rutina, el de la supervivencia y el de la trascendencia. (Litwin, E. y otros, 2007).

\section{Referencias Bibliográficas}

- Bruner, J. (2003). Fábrica de Historias. Buenos Aires, Fondo de Cultura Económica.

- Bruner, J. (1997). La educación, puerta de la cultura. Madrid, Visor.

- Fenstermacher, G. (1989). "Tres aspectos de la filosofía de la investigación sobre la enseñanza", en Wittrock, M. La investigación de la enseñanza I. Barcelona, Paidós.

- Greene, M. (2005). Liberar la imaginación. Ensayos sobre educación, arte y cambio social. Barcelona, Gráo.

- Hargreaves, A. (1996). Profesorado, cultura y postmodernidad. (Cambian los tiempos, cambia el profesorado). Madrid, Morata.

- Inhelder, B (1984) Psicología del niño. Decimotercera edición. Madrid, Morata.

- Martín, D. (2001). "Particularidades de las instituciones del Nivel Medio. El caso de las escuelas técnicas", en Actas Pedagógicas, Año II. Na 1. U.N.Co.

- Mercer, N. (1995). La construcción guiada del conocimiento. El habla de profesores y alumnos. Barcelona, Paidós.

- $\quad$ Litwin, E., Palou de Maté, M.; Calvet, M.; Chrobak, E., Herrera, M., Pastor, L. \& Sobrino, M. (2006). Evaluar la propia enseñanza. Los escenarios de la escuela media. U.N.Co, Publifadecs.

- Palou de Maté, C. (2003). "Evaluar para enseñar y evaluar para acreditar", en La enseñanza y la evaluación. Una propuesta para matemática y lengua. Buenos Aires, GEEMA - C.E.Di.Co. U.N.Co.

- Pérez Gómez, A. (1998). La cultura escolar en la sociedad neoliberal. Madrid, Morata.

- Perrenoud, P. (1990). La construcción del éxito y el fracaso escolar. Madrid, Morata. 